\title{
Вакцинация от COVID-19: модели поведенческой экономики
}

\author{
Мельникова О.А., Мельников М.Ю. \\ Уральский государственный медицинский университет, \\ Россия, 620028, г. Екатеринбург, ул. Репина, 3 \\ Email: newfarmacia@mail.ru
}

\begin{abstract}
Аннотация. Важную роль в жизни современного человека играет проблема выбора. В настоящее время поведенческая экономика рассматривается как качественно-положительная составляющая, применяемая к сопутствующей традиционной теории поведения потребителей. Это связано с тем, что с помощью поведенческой экономики можно объяснить различные противоречивые способы совершения определённых действий, которые лежат в основе выбора и суждений людей и не могут быть объяснены классической экономикой. В данной статье поведенческая экономика хорошо применяется к фармацевтической области, а именно к вопросам, связанным с вакцинацией в условиях COVID-19. Однако несмотря на то, что фармацевтическая область является очень благодатной почвой для исследований в области поведенческой экономики, исследования поведения потребителя в условиях коронавирусной инфекции практически отсутствуют. В данной работе мы постарались рассмотреть различные точки зрения, связанные с применением подходов поведенческой экономики и принятию решений о вакцинации. Исследования проводились с помощью эконометрики и математических методов. В связи с этим целью настоящего исследования является объяснение поведения потребителя по вопросам, связанным с вакцинацией, и построение математической модели. В данной статье рассмотрены ответы на такие вопросы, как поведенческая экономика может предотвратить распространение пандемии. Позволяет понять, почему соблюдение ограничений утомительно для потребителя и какую полезность с точки зрения потребителей может принести вакцинация. В работе предоставлен широкий обзор, показывающий, как стратегии поведенческой экономики могут влиять и реализовываться в различных областях фармацевтической области. В результате исследования были выявлены основные тенденции, влияющие на поведение потребителя, при вакцинации и описана математическая модель, выбора потребителя, связанная с вакцинацией от COVID-19. Полученные результаты вносят вклад в развитие поведенческой экономики в экстремальных ситуациях.
\end{abstract}

Ключевые слова: поведенческая экономика, математическая модель, вакцинация, потребитель.

Для цитирования: Мельникова О.А., Мельников М.Ю. 2021. Вакцинация от COVID-19: модели поведенческой экономики. Экономика. Информатика, 48 (3): 487-494. DOI 10.52575/2687-0932-202148-3-487-494.

\section{Vaccination against COVID-19: behavioral economics models}

\author{
Olga A. Melnikova, Mikhail Yu. Melnikov \\ Ural State Medical University \\ 3 Repin St, Yekaterinburg, 620028, Russia \\ Email: newfarmacia@mail.ru
}

\begin{abstract}
The problem of choice plays an important role in the life of a modern person. Currently, behavioral economics is viewed as a qualitatively positive component applied to the accompanying traditional theory of consumer behavior. This is due to the fact that with the help of behavioral economics, it is possible to explain various contradictory ways of performing certain actions that underlie the choices and judgments of people and cannot be explained by classical economics. In this article, behavioral economics applies well to the pharmaceutical field, specifically to issues related to vaccination in the context of COVID-19. However, despite the fact that the pharmaceutical field is a very fertile ground for research in the field of behavioral economics,
\end{abstract}


research on consumer behavior in the context of coronavirus infection is practically absent. In this paper, we have tried to consider the different perspectives associated with the application of behavioral economics approaches and making decisions about vaccination. The research was carried out using econometrics and mathematical methods. In this regard, the purpose of this study is to explain consumer behavior on issues related to vaccination and to build a mathematical model. This article looks at answers to questions such as behavioral economics can prevent the spread of a pandemic. Provides insight into why compliance is tedious for the consumer and what benefits vaccination can bring to consumers. The paper provides a broad overview showing how behavioral economics strategies can influence and be implemented in various areas of the pharmaceutical field. As a result of the study, the main trends affecting consumer behavior during vaccination were identified and a mathematical model of consumer choice associated with vaccination against COVID-19 was described. The results obtained contribute to the development of behavioral economics in extreme situations.

Keywords: behavioral economics, mathematical model, vaccination, consumer.

For citation: Melnikova O.A., Melnikov M.Yu. 2021. Vaccination against COVID-19: behavioral economics models. Economics. Information technologies, 48 (3): 487-494 (in Russian). DOI 10.52575/2687-0932-202148-3-487-494.

\section{Введение}

Поведенческая экономика - это новое направление в области экономики, которое изучает влияние множества факторов (социальных, когнитивных, эмоциональных). Данное направление экономических исследований выходит за область традиционной экономической теории, поскольку раскрывает процессы и механизмы, которые влияют на поведение человека и принятие им решений о совершении той или иной покупки. Поведенческая экономика основана на таких известных науках, как психология, политика, социология [Ариели, 2013]. Однако наиболее тесно она связана с маркетингом [Laibson, 2015; Chetty, 2015]

Фармацевтическая отрасль постоянно развивается и представляет собой интересную нишу для исследования поведенческой экономики. В связи с этим необходимо провести исследования, целью которых является изучение влияния поведенческой экономики в период Covid-19 [Решетнева, Мельникова, 2019]. Актуальность данной статьи также подтверждается тем, что стратегии поведенческой экономики вполне могут внедряться на различных уровнях лекарственного обеспечения и в разнообразных аптечных организациях [Важенина, Мельникова, 2019], способствовать стимулированию эффективного поведения потребителей [Ariely et al., 2003].

В основе традиционной теории потребительского поведения лежит понятие человек экономический (или человек рациональный). Он является разумным существом и всегда стремится максимально извлечь прибыль из своей деятельности и осуществляет выбор экономических результатов своей деятельности. В такой концепции «человек рациональный» представляет собой человека с рациональным началом и твердым расчётом [Blaug, 1995]. Он не поддается влиянию эмоций, обладает определенной силой воли и категоричностью, он тщательно просчитывает все варианты и тщательно взвешивает все плюсы и минусы от тех действий, которые он хочет сделать. Из всего огромного количества информации он выбирает именно рациональное решение, сопоставляя имеющуюся информацию в соответствии со своими потребностями и предпочтениями [Frank, 2007].

Считается, что «человек рациональный» имеет уже сформированные предпочтения, которые не изменяются после того, как он приходит на рынок и делает свой выбор. Считается, что он имеет единственный интерес, который ориентирован только на материальный результат. Однако в плане такой рациональности он всё-таки представляет собой определенную ограниченность. Такая рациональность не подходит для объяснения некоторых действий и поведения потребителя. И в этом случае поведенческая экономика объясняет данный пробел [Талер, 2017]. 
В какой-то мере поведенческая экономика является дополнением к традиционной экономике и в какой-то степени призвана объяснить отклонение от стандартной модели, предложить какие-то новые пути для объяснения поведения потребителя. Важными понятиями поведенческой экономики является удовлетворенность от покупки, а также описание поведения людей при принятии решений, связанных с определенными рисками при выборе вариантов с известными вероятностями.

С другой стороны, на поведенческую экономику влияет эвристический (информированный) поиск, который представляет собой определенную совокупность приемов и методов. Данный поиск облегчает и упрощает решение различных познавательных, конструктивных и практических задач. Однако применение этой теории также может привести к активному искажению информации и определенным систематическим ошибкам при моделировании поведения потребителя [McAuley, 2010].

Теория дуального процесса мышления сравнивает выбор с двух сторон. С одной стороны - это логика, факты, рациональность, с другой стороны - это интуиция, чувство, иррациональность, т. е. это две противоположные системы. В зависимости от типа покупки эти две системы взаимодействуют друг с другом и представляют собой определенную комбинацию при принятии решения. В этой теории становится понятно, почему люди, у которых более сильная эмоциональная и импульсивная системы, чем рациональная, очень часто принимают иррациональное решение, которое не поддается объяснению [Camerer, Loewenstein, Rabin, 2014].

В теории перспектив люди часто оценивают выбор между альтернативами, связанными с рисками и выбором, имеющим определенность. Согласно данной теории люди относятся к возможным потерям с большей осторожностью, чем к возможной выгоде в случае приобретения того или иного товара. При осуществлении выбора в данной теории на потребителя будет влиять момент, связанный с упущенной выгодой. В результате того, что потребитель будет делать только один вариант выбора из возможных вариантов. Это является минусом данной теории и заставляет поставщиков представлять на рынок наилучшие возможные альтернативы с определённым обоснованием качества своего продукта, чтобы облегчить осознание правильного выбора потребителя.

Таким образом, поведенческая экономика признаёт, что потребители не всегда обладают полностью рациональным мышлением. Они стремятся удовлетворить свои потребности, реализовать свои предпочтения согласно разным идеям [Douma, Schreuder, 2012].

Пандемия COVID-19, вероятно, изменила почти все аспекты нашей деятельности - рынок труда, образование, человеческие отношения, в том числе и поведенческий выбор. Введены нисходящие ограничения и ограничения, чтобы остановить распространение вируса и как можно скорее вернуться к «нормальному» состоянию. Однако невозможно переоценить огромное влияние индивидуального поведения и решений на развитие пандемии.

Наша способность бороться с вирусом зависит от определенного набора действий и того, как мы их выполняем. Например, избегаем скоплений людей, носим перчатки и маски для лица. И все же, как показывает поведенческая экономика, наше поведение далеко от рационального, особенно в нынешней ситуации, наполненной стагнацией, неуверенностью и страхом. Поведенческая экономика может помочь нам понять, почему нам часто трудно подчиняться строгим правилам, и почему они не всегда приносят ожидаемые результаты. Целью данного исследования является предложение модели влияния вакцинации от COVID19 на поведение потребителей, когда они сталкиваются со сложной средой выбора, выходя за рамки простых оценок рациональностей «да» / «нет». В результате появится возможность создавать более эффективные, более влиятельные на нашу избирательную рациональность действия и инициативы по борьбе с пандемией. Объектами исследования явились потребители и их поведение в условиях Covid-19. Методами исследования - построение эконометрической модели поведения потребителей. 


\section{Результаты исследования и их обсуждение}

Теорию поведенческой экономики можно успешно реализовать в принятии решения о выборе вакцинации от COVID-19. Дело в том, что фармацевтическая область является благоприятной, поскольку без лекарственных препаратов невозможно поддержание здоровья, профилактика, лечение заболеваний, выработка коллективного иммунитета.

В контексте вакцинации против COVID-19 существует несколько факторов, связанных с рисками и мерами безопасности:

1. Желание вернуться к допандемическому образу жизни, лишенному социальной дистанции, масок, удаленной работы;

2. «Пофигизим», связанный с несоблюдением ограничений и подверженность в связи с этим риску заражения;

3. Осведомленность о необходимости вакцинации, но её непроведение;

4. Эффективность (в данном случае сомнения в эффективности вакцины).

Таким образом, в поведение потребителя в условиях пандемии COVID-19 вносятся элементы иррациональности.

Математически модель выбора может быть описана уравнением [Abaluck, Gruber, 2011] и адаптирована к ситуации COVID-19:

$$
U_{i j}=P j * \alpha+E \beta 1+\sigma_{i j}^{2} * \beta_{2}+c_{j} * \beta_{3}+Q_{j} * \beta_{4}+e_{i j}(1) .
$$

Здесь $U_{i j}$ - это общая полезность, обусловленная вакцинацией от Covid-19 ji-м потребителем, J - количество доступных вакцин, зарегистрированных на рынке, $\mathrm{Pj}$ полезность от единичной вакцинации, $\mathrm{E}$ - ожидаемые расходы для государства на производство вакцины і по полезности $\mathrm{Pj}, \sigma_{i j}^{2}$ - дисперсия расходов, $c_{j}$ - вектор финансовых характеристик $\mathrm{Pj}$, влияющих на ожидаемые расходы, $Q_{j}$ - вектор показателей качества $\mathrm{P}$ (например, фирмы производители), $e_{i j}$-предполагает экстремальное значение для превращения уравнения в полиномиальную формулу.

В случае если потребители безразличны к мерам предосторожности и проведению вакцинации, то $\alpha=\beta_{1}<0$. Тут важно понимать, что потребители во время пандемии должны нести ответственность за своё поведение - должны мыть руки, носить маски, сохранять социальную дистанцию, вакцинироваться для того, чтобы выработать коллективный иммунитет и не заболеть. Однако все эти преимущества неопределённы и размыты. Потребителя беспокоят вопросы: «Если я однажды не вымыл руки и не надел маску, не заболею ли я?», «Сколько раз необходимо вакцинироваться, чтобы получить иммунитет (пользу) и не заболеть?». Поскольку видимая польза не происходит слишком быстро, а мы всегда интуитивно настроены на быстрый эффект, не хотим нести дополнительные расходы и соблюдать наложенные ограничения. Таким образом, полезность от единичной вакцинации $(\mathrm{Pj})$ и ожидаемые расходы (Е) для государства на производство вакцины в условиях риска являются безразличными для потребителей. Также $\beta_{3}=0$, поскольку потребители безразлично относятся к финансовым характеристикам вакцины и её качеству, которые приводят к одинаковым $\mathrm{E}$, а также $\sigma_{i j}^{2}$.

Можно ожидать, что $\beta_{2}<0$ при условии, что потребители не склонны рисковать и являются законопослушными гражданами, понимающими важность вакцинации. Конечно, в этом случае таких рациональных потребителей могут интересовать вопросы, связанные с качеством вакцин $\beta_{4}>0$.

Если $|\alpha|>>\left|\beta_{1}\right|$, то потребитель является очень чувствительным к полезности от вакцинации. Если $\beta_{3} \neq 0$, можно считать, что потребителей действительно будет волновать стоимость вакцин (вернее отечественное или зарубежное её производство), с помощью которых будет достигаться ожидаемый эффект от вакцинации и выработка коллективного 
иммунитета. В этом случае, $\beta_{2}$ также может быть меньше нуля $\left(\beta_{2}<0\right)$, но незначительно, что связано с незначительным аспектами, связанными с усталостью потребителей от принятия решений, гарантирующих им правильный ход событий в будущем.

Например, по мере того как возрастает сложность решений, растет и наша усталость. В случае эпидемии COVID-19 выбор, который мы должны сделать, представляет собой сложную задачу. В новой реальности, и решения касаются не только соблюдения ограничений («рискуете ли вы, если встречаетесь с друзьями?»), но и многих других аспектов нашей жизни. Из-за существования совершенно новой и ранее неизвестной реальности мы вынуждены делать совершенно новый выбор. В случае профессиональной работы, например, помимо решений, относящихся к ее сути, мы должны решить все второстепенные вопросы, связанные с ее удаленным характером. Соответственно и вопросы, связанные с вакцинацией и её рисками тяжелого течения, но позволяющие в будущем после выработки иммунитета убрать аспекты, связанные с удаленной работой и перейти к полноценному общению, также рассматриваются потребителями.

Решения, которые мы принимаем в этой совершенно новой реальности, часто имеют неопределенные или неизвестные последствия. Таким образом, они более сложны и, следовательно, более утомительны, и чем больше мы устаем, тем худший выбор мы сделаем в отношении своего здоровья, работы и всех других аспектов нашей жизни. Более того, импульсивные действия, вызванные усталостью от принятия решений, по определению более рискованны и менее продуманы, поэтому могут представлять потенциальную угрозу для нашего здоровья.

Принимать решения - очень утомительно, поскольку требуется постоянный самоконтроль. А в период пандемии все устали от самоконтроля. Такой эффект можно называть истощением воли. Это связано с тем, что при самоконтроле требуются большие умственные ресурсы, которые интенсивно используются, а любая эмоционально требовательная ситуация их истощает. Истощение воли ведет к менее сдержанным и «разумным» решениям и действиям, поскольку контролировать себя утомительно, потребитель в какой-то момент теряет над собой контроль.

В период пандемии потеря контроля и совершение импульсивных поступков по несоблюдению коронавирусных ограничений приводят к волнам заболеваний. Т. е. истощение воли в переводе в контексте пандемии - это потеря самоконтроля, который может быть связан с отказом от ношения маски, мытья рук или отказом от встречи с друзьями, утрата нашей способности ограничивать себя.

Тем не менее необходимо понимать, что данная математическая модель не учитывает деление на рациональных и нерациональных потребителей. В этом случае, может быть два вида моделей [Houser, Keane, McCabe, 2004].

Для рациональных, принимающих решение на основе полезности вакцинации:

$$
U_{i j}=(P j+E) * \beta_{1 i}+\sigma_{i j}^{2} * \beta_{2 i}+Q_{i j} * \beta_{4 i}+e_{i j}(2) .
$$

И потребителей, которые сомневаются в возможном эффекте от вакцинации, задумываясь о её побочном действии:

$$
U_{i j}=P_{j} \alpha_{i}+E_{i j} * \beta_{1 i}+\sigma_{i j}^{2} * \beta_{2 i}+c_{i j} * \beta_{3 i}+Q_{i j} * \beta_{4 i}+e_{i j}(3) .
$$

В уравнении 2 имеются ограничения $\alpha=\beta_{1}, \beta_{3}=0$. Но принципиальное отличие состоит в том, что мы налагаем эти ограничения на индивидуальном уровне, а не на общие параметры, оцениваемые на основе объединенных данных. В отличие от уравнения (2) уравнение (3) не накладывает этих ограничений. Наконец, случайный член $e_{i j}$ занимает крайнее значение в уравнениях обоих типов. Таким образом, в зависимости от типа поведения человека и параметров его предпочтений при вакцинации, мы получаем простую полиномиальную логит-модель. Данная модель, на взгляд авторов, может быть использована для прогнозирования вероятности вакцинации путём его сравнения с логистической кривой. 
Таким образом, поведенческая экономика позволяет не только понять, почему соблюдение ограничений утомительно для нас, но и позволяет описать математически целый ряд действий, которые можно использовать для влияния на общество, учитывая неполную рациональность людей. Отдельные лица и группы потребителей могут влиять не только на разрешение или запрещение, но и формировать определённую информационную среду по проблеме вакцинации, побуждая других потребителей сделать правильный для них вывод. А полезность от проведения вакцинации может быть описана предложенными моделями.

\section{Заключение}

В заключение стоит отметить, что в данной статье рассматривается поведенческая экономика применительно к вакцинации и поведению потребителей в условиях COVID-19. Приводится объяснение поведения людей благодаря новым взглядам с точки зрения экономики, психологии и когнитивных функций. Подходы, изложенные в данной статье, также, как и стратегии поведенческой экономики могут быть реализованы в различных областях, связанных c иммунопрофилактикой $\mathrm{c}$ целью эффективного понимания поведения потребителей. Поведенческая экономика не является самостоятельной отраслью и не должна рассматриваться отдельно от традиционной экономической теории, а скорее всего будет представлять определенный вклад в арсенале современной экономики. Поведенческая экономика может быть очень полезной в фармацевтической области, поскольку она является эффективным поведенческим инструментом, позволяющим понять поведение потребителя и выявить теоретические последствия, особенности политики государства, направленной на поддержание и сохранение здоровья людей.

\section{Список литературы}

1. Ариели Д. 2013. Поведенческая экономика. Почему люди ведут себя иррационально и как заработать на этом. М.: Манн, Иванов и Фербер, 296 с.

2. Важенина Д.А., Мельникова О.А. 2019. Изучение потребительских предпочтений при выборе препаратов для купирования головной боли. В сборнике: Современная мировая экономика: проблемы и перспективы в эпоху развития цифровых технологий и биотехнологии. Сборник научных статей по итогам работы девятого международного круглого стола, 206-208.

3. Решетнева А.С., Мельникова О.А. 2019. Маркетинговые исследования посетителей аптек г. Екатеринбурга. В сборнике: Молодежь - практическому здравоохранению XIII Всероссийская с международным участием научная конференция студентов и молодых ученых-медиков, 360-362.

4. Талер Р. 2017. Новая поведенческая экономика. Почему люди нарушают правила традиционной экономики и как на этом заработать. М.: Эксмо, 368 с.

5. Abaluck J., Gruber J. 2011. Choice inconsistencies among the elderly: Evidence from plan choice in the medicare Part D program. American Economic Review, 101 (4): 1180-1210.

6. Ariely D., Loewenstein G., Prelec D. 2003. "Coherent arbitrariness": stable demand curves without stable preferences. Quarterly Journal of Economics, 118 (1): 73-105.

7. Blaug M. 1995. Economic theory in retrospect. Cambridge University press, 633 p.

8. Camerer C.F., Loewenstein G., Rabin M. 2004. Advances in behavioral economics. Russell Sage Foundation, $51 \mathrm{p}$.

9. Chetty R. 2015. Behavioral economics and public policy: a pragmatic perspective. The American Economic Review, 105: 1-33.

10.Douma S.W., Schreuder H. 2012. Economic approaches to organizations. Pearson Education, 282 p.

11.Elrod T., Keane M. 1995. A factor-analytic probit model for representing the market structure in panel data. Journal of Marketing Research, 32 (1): 1-16.

12.Erdem T., Keane M. 1996. Decision making under uncertainty: Capturing dynamic brand choice processes in turbulent consumer goods markets. Marketing Science, 15 (1): 1-20.

13.Erdem T., Keane M., Sun B. 2008. A dynamic model of brand choice when price and advertising signal product quality. Marketing Science, 27 (6): 1111-1125.

14.Fang H., Keane M., Silverman D. 2008. Sources of advantageous selection: Evidence from the medigap insurance market. Journal of Political Economy, 116 (2): 303-350. 
15.Frank R. 2007. Behavioral economics and health economics, Diamond, Vartiainen (Eds.), Behavioral Economics and Its Applications, Princeton Univ. Press, Princeton, NJ, 195-234.

16. Houser D., Keane M., McCabe K. 2004. Behavior in a dynamic decision problem: an analysis of experimental evidence using a Bayesian type classification algorithm. Econometrica, 72: 781-822.

17.McAuley I. 2010. When does behavioral economics really matter? Paper to accompany presentation to behavioral economics stream at Australian Economic Forum.

18.Laibson D., List J.A. 2015. Principles of (behavioral) economics. The American Economic Review, 105: 385-390.

\section{References}

1. Ariyeli D. 2013. Povedencheskaya ekonomika. Pochemu lyudi vedut sebya irratsional'no i kak zarabotat' na etom [Behavioral economics. Why do people behave irrationally and how to make money on it?] M.: Mann, Ivanov i Ferber, 296 p. (in Russian)

2. Vazhenina D.A., Mel'nikova O.A. 2019. Izucheniye potrebitel'skikh predpochteniy pri vybore preparatov dlya kupirovaniya golovnoy boli [Study of consumer preferences when choosing medications for headache relief]. V sbornike: Sovremennaya mirovaya ekonomika: problemy i perspektivy v epokhu razvitiya tsifrovykh tekhnologiy i biotekhnologii Sbornik nauchnykh statey po itogam raboty devyatogo mezhdunarodnogo kruglogo stola, 206-208. (in Russian)

3. Reshetneva A.S., Mel'nikova O.A. 2019. Marketingovyye issledovaniya posetiteley aptek g. Yekaterinburga [Marketing research of visitors to pharmacies in Yekaterinburg]. V sbornike: Molodezh' prakticheskomu zdravookhraneniyu XIII Vserossiyskaya s mezhdunarodnym uchastiyem nauchnaya konferentsiya studentov i molodykh uchenykh-medikov, 360-362. (in Russian)

4. Taler R. 2017. Novaya povedencheskaya ekonomika. Pochemu lyudi narushayut pravila traditsionnoy ekonomiki i kak na etom zarabotat' [The new behavioral economy. Why do people violate the rules of the traditional economy and how to make money on it]. M.: Eksmo, 368 p. (in Russian)

5. Abaluck J., Gruber J. 2011. Choice inconsistencies among the elderly: Evidence from plan choice in the medicare Part D program. American Economic Review, 101 (4): 1180-1210.

6. Ariely D., Loewenstein G., Prelec D. 2003. "Coherent arbitrariness": stable demand curves without stable preferences. Quarterly Journal of Economics, 118 (1): 73-105.

7. Blaug M. 1995. Economic theory in retrospect. Cambridge University press, $633 \mathrm{p}$.

8. Camerer C.F., Loewenstein G., Rabin M. 2004. Advances in behavioral economics. Russell Sage Foundation, $51 \mathrm{p}$.

9. Chetty R. 2015. Behavioral economics and public policy: a pragmatic perspective. The American Economic Review, 105: 1-33.

10. Douma S.W., Schreuder H. 2012. Economic approaches to organizations. Pearson Education, 282 p.

11.Elrod T., Keane M. 1995. A factor-analytic probit model for representing the market structure in panel data. Journal of Marketing Research, 32 (1): 1-16.

12.Erdem T., Keane M. 1996. Decision making under uncertainty: Capturing dynamic brand choice processes in turbulent consumer goods markets. Marketing Science, 15 (1): 1-20.

13.Erdem T., Keane M., Sun B. 2008. A dynamic model of brand choice when price and advertising signal product quality. Marketing Science, 27(6): 1111-1125.

14.Fang H., Keane M., Silverman D. 2008. Sources of advantageous selection: Evidence from the medigap insurance market. Journal of Political Economy, 116(2): 303-350.

15.Frank R. 2007. Behavioral economics and health economics, Diamond, Vartiainen (Eds.), Behavioral Economics and Its Applications, Princeton Univ. Press, Princeton, NJ, 195-234.

16. Houser D., Keane M., McCabe K. 2004. Behavior in a dynamic decision problem: an analysis of experimental evidence using a Bayesian type classification algorithm. Econometrica, 72: 781-822.

17.McAuley I. 2010. When does behavioral economics really matter? Paper to accompany presentation to behavioral economics stream at Australian Economic Forum.

18.Laibson D., List J.A. 2015. Principles of (behavioral) economics. The American Economic Review, 105: 385-390.

Конфликт интересов: о потенциальном конфликте интересов не сообщалось.

Conflict of interest: no potential conflict of interest related to this article was reported. 
ИНФОРМАЦИЯ ОБ АВТОРАХ

Мельникова Ольга Александровна, доктор фармацевтических наук, доцент, профессор кафедры управления и экономики фармации, Уральский государственный медицинский университет, г. Екатеринбург, Россия

Мельников Михаил Юрьевич, кандидат химических наук, доцент кафедры фармации и химии, Уральский государственный медицинский университет, г. Екатеринбург, Россия

\section{INFORMATION ABOUT THE AUTHORS}

Olga A. Melnikova, Doctor of Pharmaceutical Sciences, Associate Professor, Professor of the Department of Management and Economics of Pharmacy, Ural State Medical University, Yekaterinburg, Russia

Mikhail Yu. Melnikov, Candidate of Chemical Sciences, Associate Professor of the Department of Pharmacy and Chemistry, Ural State Medical University, Yekaterinburg, Russia 